\title{
Martine Sagaert - Peter Schnyder, André Gide. L'écriture vive
}

\section{Gian Luigi Di Bernardini}

\section{(2) OpenEdition}

\section{Journals}

\section{Edizione digitale}

URL: http://journals.openedition.org/studifrancesi/7014

DOI: $10.4000 /$ studifrancesi.7014

ISSN: 2421-5856

\section{Editore}

Rosenberg \& Sellier

\section{Edizione cartacea}

Data di pubblicazione: 1 septembre 2010

Paginazione: 402

ISSN: 0039-2944

\section{Notizia bibliografica digitale}

Gian Luigi Di Bernardini, «Martine Sagaert - Peter Schnyder, André Gide. L'écriture vive», Studi Francesi [Online], 161 (LIV | II) | 2010, online dal 30 novembre 2015, consultato il 14 janvier 2021. URL: http:// journals.openedition.org/studifrancesi/7014; DOI: https://doi.org/10.4000/studifrancesi.7014

Questo documento è stato generato automaticamente il 14 janvier 2021.

\section{(c) $(7)$}

Studi Francesi è distribuita con Licenza Creative Commons Attribuzione - Non commerciale - Non opere derivate 4.0 Internazionale. 


\title{
Martine Sagaert - Peter Schnyder, André Gide. L'écriture vive
}

\author{
Gian Luigi Di Bernardini
}

\section{NOTIZIA}

MARTINE SAGAERT - PETER SCHNYDER, André Gide. L'écriture vive, Bordeaux, Presses

Universitaires de Bordeaux, 2008, pp. 163, con DVD.

1 Gide dedicava il suo Journal des Faux-Monnayeurs, diario della genesi del suo "unico" romanzo, «à ceux que les questions de métier intéressent» (p. 9). Sarebbe sufficiente questa dichiarazione per spiegare la progressiva diffusione di edizioni genetiche dell'opera gidiana la quale si nutre di un'attenzione notevole al divenire della scrittura nel momento stesso del suo farsi, tanto da essere rappresentata nel testo tramite la figura della mise en abyme.

2 Dopo un primo capitolo introduttivo, che permette al lettore di comprendere le caratteristiche principali di un'edizione genetica e di formarsi un primo orientamento sulla situazione delle edizioni a oggi esistenti dei testi gidiani pubblicati (comprese quelle critiche e le poche genetiche già prodotte), Sagaert e Schnyder puntualizzano alcune delle loro scelte. Tra queste va segnalata la loro attenzione all'altissimo quoziente informativo apportato da uno studio delle bozze a cui Gide, secondo varie testimonianze, era particolarmente sensibile. Il dossier genetico non si limita, quindi, a prendere in considerazione il solo manoscritto, ma anche le bozze stampate e il lavoro di correzione autoriale.

3 I testi esaminati vengono raccolti dai curatori in due grandi rubriche, una relativa a questioni interne che raccoglie testi personali e intimi come il Journal e Ainsi soit-il, l'altra a questioni esterne, concernente il rapporto con paesi e lingue straniere, come nel caso del Retour de l'U.R.S.S. e di alcune traduzioni, tra cui va segnalata quella di Hamlet di Shakespeare. 
Il DVD-rom contiene diverse sezioni e numerose informazioni. La «Médiathèque» raccoglie materiali diversi, alcuni più generali come una biografia strettamente cronologica, i luoghi di conservazione dei manoscritti gidiani, una bibliografia (maggiormente incentrata sui testi e le edizioni critiche e che si limita ai soli testi critici di base), un'iconografia (contenente alcune fotografie più note e altre nuove), un'antologia di alcune frasi gidiane significative, alcuni passi tratti dal film di JeanPierre Prévost Gide, un petit air de famille. Nella sezione «Les Travaux de Gide», il lettore accede ai documenti veri e propri, organizzati secondo il doppio principio interno/ esterno che abbiamo già visto. Per il primo volet, si può vedere il Journal, di cui Sagaert propone quattro quaderni, scritti tra il 1926 e il 1928 e non inseriti nemmeno nella seconda edizione del Journal pubblicata nel 1996-1997. Sono visibili inoltre i manoscritti di Ainsi soit-il, appartenenti a una collezione privata. Riguardo al secondo volet, è visibile il manoscritto inedito della traduzione che Gide tentò nel 1922 dell'Amleto di Shakespeare, in particolare degli atti I e II (scene 1 e 2), versi 1-86. Vi sono inoltre le traduzioni (iniziate, ma non completate) della corrispondenza tra lo scrittore Friedrich Hebbel e Elise Lensing, incentrata sul soggiorno parigino dello scrittore tedesco.

5 Nella sezione «Les Manuscrits des autres» si può avere un saggio dell'interesse di Gide per l'opera in fase di costruzione di altri scrittori, interesse testimoniato dalla presenza dei manoscritti di Montaigne, Rilke e Charles-Louis Philippe. Nella sezione «Laboratoire de la création» si trovano delle foto ritraenti i luoghi in cui Gide era solito produrre e alcuni cenni ai supporti materiali usati dall'autore.

Dans L'Euvre à son estuaire, infine, alcuni video si soffermano su diversi aspetti materiali della scrittura gidiana, compresa l'analisi della pagina manoscritta contenente le ultime righe scritte da Gide. 